American medical associations. These recommendations stress the importance

\title{
A protocol for the reduction of surgical errors
}

\section{S Bann, A Darzi}

\section{The importance of reporting errors in "real time"}

M edical errors make headline news. The headlines will always emphasise the human suffering associated with medical error, but the prevention of such errors comes as the result of detailed analysis of their circumstances. One area of particular concern to surgeons is wrong site/wrong side surgery. Although its occurrence is rare, it is potentially avoidable. A notable recent case in the UK was that of a patient who died from renal failure after the removal of his healthy kidney and not his diseased one. Worldwide systems should be in place to prevent this occurring, but the data revealing the extent of the problem have not been readily available.

In the past there has been little opportunity to understand the cause of errors as such events-although rarewere often not reported and not collated with other similar events, preventing their repeated recurrence. Not surprisingly, doctors were often reluctant to disclose such errors or "near misses". But attitudes and practices are changing. In the USA the Joint Commission on Accreditation of Healthcare Organizations (JCAHO; www.jcaho.org; see box 1) has shown that, by the voluntary reporting of serious errors to a central body, these events can be analysed. This has revealed not only the relative rarity of wrong site/wrong side surgery, but has also allowed the pooling of data and their analysis, permitting guidelines to be drawn up to prevent their potential recurrence.

When retrospective studies are undertaken, these suggest that at least $5-10 \%$ of hospital admissions will suffer an adverse event. ${ }^{1-5}$ By inference and extrapolation, this would imply that 1.3 million people in the USA are harmed annually and in the UK the figure would be over $400000 .{ }^{1}$ Within these figures, surgery is rated the highest risk factor for all adverse events. ${ }^{6}$ Root cause analysis of medical errors leading to adverse events reveals them to be multifactorial. ${ }^{7}$ In the case of wrong site surgery, this will involve a breakdown in communication between members of the surgical team and the patient and his/her family. Contributory factors will include problems with the routine process of "site marking" (that is, marking the site of the operation with indelible ink before anaesthesia), failure in the verification of a preoperative checklist, incomplete patient assessment, missing patient notes, distraction, and various other organisational issues. However, a major problem with these retrospective studies is that it is difficult to obtain "real time" information regarding the circumstances.

In the USA the JCAHO has been supporting a voluntary reporting system since 1996 that potentially allows clinicians to report errors as they occur; it also examines other sources such as the media. This information is collated and examined and reported as a periodic newsletter called Sentinel Event Alerts which describes common causes and suggests steps for elimination and prevention of their recurrence. This includes not just wrong site surgery but also other areas such as medication errors, blood transfusions, and postoperative complications.

For wrong site/wrong side surgery the JCAHO has so far reported 150 cases, with root cause analysis available for 126: $75 \%$ were on the wrong body part or site, $13 \%$ were for wrong patient surgery, and $11 \%$ for the wrong surgical procedure. Over $80 \%$ of these incidents were self-reported, with the remainder arising mainly from patient complaints. In New York State, where a mandatory system for reporting is in place, there were 46 cases in 2 years, ${ }^{8}$ which suggests that these voluntary figures could be a vast underestimate. A recent survey of hand surgeons in the USA found that at least $20 \%$ of them had undertaken wrong site surgery at least once in their career, and a further $16 \%$ had only avoided this at the time of incision. ${ }^{9}$ This reinforces the necessity for prospective reporting.

From their analysis of prospective reporting of wrong site surgery, the JCAHO has issued the "Universal protocol for preventing wrong site, wrong procedure, wrong person surgery" which has been endorsed by the major of risk reduction strategies including checking and rechecking. The marking of the surgical site should involve the patient; the use of verification checklists and the availability of the appropriate documents such as notes and $x$ rays; verification by the patient of the site in the operating theatre complex; and the monitoring of these processes; a final time out is suggested before starting the procedure with active communication involving the whole operative team. An important concept promoted by the protocol is the active involvement of the patient in the process. This requires the clinical team to involve patients in decisions about their care.

In the UK this could be extended to include a second patient signature on the consent form to confirm, where appropriate, that the site or area marked is correct to the best of the patient's knowledge. At present in the UK there are no nationally applied guidelines or protocols regarding site marking; local practice often requires that the nursing staff merely document that the site or side is marked, but the operating surgeon ultimately takes responsibility and this potentially provides a source of individual error.

It is clearly important that, if errors occur, lessons should be learnt to prevent their recurrence. The JCAHO approach has shown that, with voluntary collection, it is possible to capture details of errors in "real time", although this may not reflect the true incidence of errors. Analysis of the information provided by the clinicians reporting the errors can aid the development of guidelines aimed at preventing their recurrence. In the UK the National Patient Safety Agency (www.npsa. nhs.uk) is developing a national reporting system for patient safety incidents which is being implemented in England and Wales-the National Reporting and Learning System (NRLS). This has been trialled by 39 health service organisations and is in the process of being rolled out nationally. The aim is to identify recurrent patterns of behaviour and practice associated with errors and to feed this back to ensure safer care. It is envisaged that, by encouraging the reporting of errors, their number will decline, paralleling the airline industry. However, it should be recognised that, despite increasing technical reliability, human error can never be totally removed..$^{10}$

The reporting of medical errors in "real time" will reveal a truer picture of their frequency. This offers the potential of providing solutions that will reduce the risk or even prevent their occurrence 


\section{Box 1 JCAHO}

The JCAHO, founded over 50 years ago, has a declared mission to improve continuously the safety and quality of care provided to the public. A major role is to identify the cause of harmful errors and facilitate their reduction through analysis, reporting, implementation, and monitoring of any applied policies. An effective reporting system is an essential prerequisite for serious event analysis and needs to be within a framework that allows the information to be legally protected from disclosure so that data can be seen as Sentinel Event Alerts. Data from the analysis of reported serious events are used as the foundation for the formulation and implementation of safety and quality guidelines.

in identical circumstances. Patient involvement seems simple but is, in fact, a major shift in emphasis. Despite evidence from the airline industry where safety is also paramount, the junior staff have clear instructions to challenge their seniors in situations of potential error whereas in surgery the likelihood of the junior surgeons and staff challenging their senior colleagues is much less likely. ${ }^{11}$ Indeed, in the case of the patient whose wrong kidney was removed, it is reported that a medical student present in the operating theatre did suggest wrong side surgery. Any guidelines issued must therefore provide backing for issues that may arise from this challenge.

The JCAHO has shown the way with the reporting and analysis of these incidents. We must ask whether there is any reason why the UK should not adopt the protocol that has emerged from their experience.

Qual Saf Health Care 2004;13:162-163.

doi: 10.1136/qshc.2003.009431

\section{Authors' affiliations}

S Bann, Department of Surgery, Chelsea and Westminster Hospital, London SW10 9NH, UK

A Darzi, Department of Surgical Oncology and Technology, Imperial College of Science, Technology and Medicine, St Mary's Hospital, London W2 INY, UK

Correspondence to: Dr S Bann, Department of Surgery, Chelsea and Westminster Hospital, London SW10 9NH, UK; s.bann@ic.ac.uk

Conflicts of interest: none.

\section{REFERENCES}

1 Vincent C, Neale G, Woloshynowych M. Adverse events in British hospitals: preliminary retrospective record review. BMJ 2001;322:517-9.

2 Brennan TA, Leape LL, Laird NM, et al. Incidence of adverse events and negligence in hospitalized patients. Results of the Harvard Medical Practice Study I. N Engl J Med 1991;324:370-6.

3 Leape LL, Brennan TA, Laird N, Lawthers AG, Localio AR, Barnes BA, et al. The nature of adverse events in hospitalized patients. Results of the Harvard Medical Practice Study II. NEngl J Med 1991;324:377-84.

4 Wilson RM, Runciman WB, Gibberd RW, et al. The Quality in Australian Health Care Study. Med J Aust 1995; 163:458-71.

5 Gawande AA, Thomas EJ, Zinner MJ, et al. The incidence and nature of surgical adverse events in Colorado and Utah in 1992. Surgery 1999; 126:66-75.

6 Garcia-Martin M, Lardelli-Claret P, BuenoCavanillas A, et al. Proportion of hospital deaths associated with adverse events. J Clin Epidemiol 1997;50:1319-26.

7 Vincent C, Taylor-Adams S, Chapman EJ, et al. How to investigate and analyse clinical incidents: clinical risk unit and association of litigation and risk management protocol. $B M J$ 2000;320:777-81.

8 Shojana KG, Duncan BW, McDonald KM, et al Making health care safer: a critical analysis of patient safety practices, Evidence Report/ Technology Assessment No 43. AHRQ Publication No 01-E058. Rockville, MD: Agency for Healthcare Research and Quality, 2001.

9 Meinberg EG, Stern PJ. Incidence of wrong-site surgery among hand surgeons. J Bone Joint Surg Am 2003;85-A: 193-7.

10 Hollnagel E. Reliability of cognition: foundations of human reliability analysis. London: Academic Press, 1993.

11 Sexton JB, Thomas EJ, Helmreich RL. Error, stress, and teamwork in medicine and aviation: cross sectional surveys. BMJ 2000;320:745-9.

professional accountability has been cast as the "black sheep" of safety improvement. Undeveloped systems of professional accountability, inadequate support from professional bodies for professional regulation, inadequate understanding of public interest, and inadequate rules for reporting serious misconduct have let this happen. This is no criticism of safety advocates whose job is to reduce patient injury: too many messages can be detrimental to success. But have we got the balance right? System theorists and industries upon which health relies for systems redesign and remedies pay a lot of attention to the role violations play in the system. Reason ${ }^{4}$ argues that, in addition to a systems approach to error management, we need effective regulators with the appropriate legislation, resources and tools. Regulators, being separate from organisations, are best placed to identify unsatisfactory work practices or conditions that workers tolerate or work around.

The perceived contest between whether individuals or bad systems cause patient injuries has confused many health professionals and managers. It is not a case of accepting one over the other. The focus on the system as the problem does not mean that 
$\mathrm{m}$ as the problem does not mean that individuals do not have to maintain competence and practice ethically or be called to account when they act unprofessionally. Accentuating the system and downplaying professional responsibility may be politically expedient to some groups, particularly those professional groups opposed to external scrutiny. But sacrificing professional accountability increases the risks to patients. The failure to urge professional responsibility concurrently with calls for a "blame free" approach to error reporting sends the public the message that the health system favours one above the other.

\section{UNDERSTANDING VIOLATIONS}

Patients making complaints about their health care are entitled to have their individual care examined to see if there has been a departure from the required standard. System issues may be the main cause. But health providers may also have cut corners and broken rules. Medical standards may have been breached and substandard care provided. Rules are broken so often in hospitalsfor example, non-compliance with a protocol such as failure to wash hands-that we have become immune to them. It is easier to blame such violations solely on the system than to require individuals to meet their professional responsibilities. Reason defines a violation as a deviation from safe operating procedures, standards, or rules. ${ }^{4} \mathrm{He}$ categories violations as routine, optimising, and necessary. The first two relate to personal characteristics while necessary violations are linked to organisational failures. Cutting corners are routine violations that thrive in work environments that rarely sanction violations or reward compliance ${ }^{4}$-for example, not following protocols, inadequate handovers, inadequate infection control, and not attending on-call requests. Optimising violations involve individuals motivated by personal goals such as greed or thrills from risk taking-for example, letting inexperienced junior staff operate without supervision when a consultant is busy with private patients, experimenting with unproven procedures, and doing inappropriate procedures. Necessary violations comprise work environments and circumstances which force workers to break rules to get the job done. Deliberate violationsthose where there is an intention to act as distinct from a violation caused through ignorance-are recognised and managed. Intentional violations do not necessarily intend a bad outcome. ${ }^{4}$ Poor understanding of professional obligations and a weak infrastructure for managing unprofessional behaviour in hospitals provide fertile ground for aberrant behaviour to flourish.

\section{LEARNING FROM THE PAST}

The main avenue of redress for patients suffering adverse events during the 1980 s and 1990s was to make a complaint. Health professions and organisations were deaf to stories of inadequate or substandard treatments and focused on the messengers (regulatory authorities, consumer groups, complaint agencies, or lawyers) as the problem. Professional accountability was the focus of these investigations, with no attention to the role played by the system. We should learn from that experience. Just as it was wrong in the past to focus only on individuals, it is equally wrong today to think that all adverse outcomes are caused by systems problems with no attention to professional duties and responsibilities.

\section{A WAY FORWARD}

In my experience as both a regulator and safety exponent, ${ }^{*}$ systems issues usually accompany breaches of professional responsibility (weak regulations, reporting requirements, or inadequate training). It depends how you look and where. A root cause analysis ${ }^{5}$ would nearly always identify systems problems and rarely individuals. Systems failures may also mitigate the level of responsibility for the individuals. Where and how professional responsibility fits into the "no blame" culture is unclear. How can we make it clearer?

Public trust requires both a redesigned health system delivering safe

*The author was the NSW Health Care Complaints Commissioner (1995-2000) and is now the Chair of the Personal and Professional Development Theme in the Faculty of Medicine University of Sydney undertaking research on quality and safety and teaching students and medical clinicians about ethical practice, quality improvement and safety. and quality health care and a strong professional ethic and accompanying accountability system. As a first step, three things should happen:

- professionalism in the workplace needs to become part of the safety agenda;

- methods for managing and responding to intentional violations by individuals in the workplace need to be debated and designed: building in sanctions for routine violations and rewards for workplace compliance is a first step;

- teaching clinicians about the inevitability of mistakes is already happening but we also need to teach them how to respond to mistakes.

Disciplinary outcomes for doctors are largely determined by peer review and focus on the actions taken after the mistake rather than the mistake itself. ${ }^{6}$ Demystifying accountability mechanisms and educating professionals about their ethical obligations will help them identity systems problems and the appropriate remedies and professional issues and their appropriate response.

\section{ACKNOWLEDGEMENT}

The author thanks Professor George Rubin and Dr Stuart Dorney for their comments on the editorial.

Qual Saf Health Care 2004;13:163-164. doi: 10.1136/qshc.2004.010959

Correspondence to: Associate Professor $M$ Walton, Faculty of Medicine, University of Sydney, Sydney 2006, Australia; mwalton@ dme.med.usyd.edu.au

\section{REFERENCES}

1 Lamb R. Open disclosure: the only approach to medical error. Qual Saf Health Care 2004; 13:3-5.

2 Millenson ML. Breaking bad news. Qual Saf Health Care 2002;11:206-7.

3 Berwick DM. Improvement, trust and the health care workforce. Qual Saf Health Care 2003;12(Suppl I):i2-6.

4 Reason JT. Managing the risks of organisationa accidents. Aldershot, UK: Ashgate Publishing, 1997

5 Joint Commission on Accreditation of Healthcare Organizations. Conducting root cause analysis in response to a sentinel event. Oakbrook Terrace, IL: Joint Commission on Accreditation of Healthcare Organizations, 1996.

6 McNeill PM, Walton M. Medical harm and the consequences of error for doctors. Med J Aust 2002; 176:22-5. 


\section{What matters more in patient care? Giving doctors shorter hours of work or a good night's sleep?}

\section{J Firth-Cozens, H Cording}

\section{Focusing only on reducing hours of work may not have the desired effect of reducing symptom levels}

$\mathrm{T}$ long hours that doctors work, and the length and quality of their sleep, have long been viewed as influencing their health and the safety of care they give. ${ }^{1}$ In 2000 it was agreed by the European Parliament that the Work-time Directive, which had limited working hours in general, should also apply to doctors in training. This will mean no more than 58 hours per week by August 2004 and eventually a maximum of 48. In Europe this has more to do with the risks towards the doctor while in the US efforts to reduce long hours have come more from their threat to patient safety. But is this focus on hours the right one?

There is certainly some evidence that a long working week will affect your health: a meta-analytical review of workers in general found small but significant positive correlations between overall health symptoms, physiological and psychological health symptoms, and hours of work. ${ }^{2}$ In Japan, where working hours are particularly long, this has been implicated in cardiovascular disorders and diabetes mellitus. ${ }^{3}$ Nevertheless, most studies show surprisingly little evidence for the relationship between hours of work and psychological well being: even when hours are long, they have little or no correlation with levels of stress or depression. ${ }^{45}$ On the other hand, a combination of the intensity of demands (rather than long hours per se) combined with low discretion over how one's work is done continues to be an influential model of the causes of job related stress. ${ }^{6}$ Doctors have consistently shown unusually high levels of stress symptoms and consider overwork to be involved in this. ${ }^{1}$ What is likely, however, is that the tiredness caused by long hours makes any potential job related stressors- such as lack of support, dealing with patient deaths, or the high cost of error in medicine-much more difficult to cope with and, in this way, stress symptoms may be a mediator between hours worked and psychological disorders such as depression or anxiety. ${ }^{7}$ Focusing only on reducing hours of work may not have the desired effect of reducing these symptom levels.

". . . it may be better to aim for ensuring good support, leadership, and teamwork . . ."

Nevertheless, doctors certainly see overwork-both long hours and a lack of support-as contributing to incidents of poor care,, 8 and evidence from a military study suggests that individual errors increase with fatigue. ' However, the same study found that, over time, the team can compensate for these errors, and so those who worked together over a longer period had higher individual error scores but lower team error scores than those working together only briefly. It seems that the relationship of hours to error is complex, mediated by such factors as the support of colleagues, including leaders, as well as the levels of control one has over one's job. ${ }^{6}$ Certainly for younger doctors, long hours of work, well managed, $^{1011}$ may be a relatively insignificant factor compared with the positive effects of being part of a team and the enjoyment of practising medicine. Rather than continuing the pursuit of a shorter working week, it may be better to aim for ensuring good support, leadership, and teamwork since good teams have healthier staff and better outcomes. $^{12} 13$

Although there is inevitably a relationship between hours of work and hours of sleep, the negative effects of sleep loss on both mood and performance have been found much more consistently than those of long hours. Sleep deprivation necessarily varies in real life studies, but most definitions involve fewer than 4 hours sleep in 24 , or frequently interrupted sleep. Most studies show that mood is lowered after a long on-call shift and young doctors report feeling more confused and less confident. ${ }^{14}$

These psychological effects are mirrored where the quality of care is considered. Errors increase with sleep loss, and data on shift work show a rise in industrial injuries on the night shift. ${ }^{15}$ In medicine, such data are rarely collected and instead a variety of cognitive tests are used as proxy measures of performance-assessing memory, concentration, alertness, and attention to detail after nights on call. Review studies ${ }^{16}$ and meta-analyses ${ }^{17}$ agree that sleep deprived people perform significantly less well than controls, particularly in terms of mood and cognitive tasks, whether proxy assessments or simulations are used. For example, surgical house officers following a weekend on call showed significant impairments in concentration and speed. ${ }^{14}$ Performance in general was highly related $(0.5-0.6)$ to the number of hours of sleep: an impairment to vigilance estimated to be close to that caused by $0.7 \mathrm{~g} / \mathrm{kg}$ alcohol which, as they point out, is near the legal UK limit. Simulations of laparoscopic surgery show that more errors occur with increasing sleep loss ${ }^{18}$ and after a night on call. ${ }^{19}$ Moreover, there is some evidence to suggest that there are deleterious effects even 2 days later. ${ }^{20}$

In the short term, an educational approach to the dangers of sleep loss and ways to improve sleep quality may be useful, alongside encouraging leaders to ensure that those deprived of sleep are not put in potentially dangerous

\section{Key messages}

- There is only a small complex relationship between hours of work and performance or mood.

- Other factors such as team relationships, leadership, and job design may matter more.

- Lack of sleep or disturbed sleep, in particular, leads to substantial decrements in performance.

- Remedying the raft of factors is likely to improve both working lives and patient safety more than focusing simply on a shorter working week. 
situations. ${ }^{10}$ The benefits of napping ${ }^{21}$ or rest periods ${ }^{22}$ in terms of subsequent performance have been reported and could be built into the working day officially rather than surreptitiously by individuals. However, it seems important that we integrate our approaches to improving working lives and patient care, ${ }^{1}$ and resist focusing only on hours or any single factor. What is needed is a systems approach towards a cultural change that uses an evidence base to address the complex factors that contribute to staff working below par, and that treats healthy alert staff as a key element of patient safety.

Qual Saf Health Care 2004;13:165-166. doi: 10.1136/qshc.2002.002725

\section{Authors' affiliations}

J Firth-Cozens, London Deanery of Postgraduate Medical and Dental Education, London WCIN 1DZ, UK

H Cording, Primary Care Development Centre, Northumbria University, Newcastle upon Tyne NE7 7XA, UK

Correspondence to: Professor J Firth-Cozens, London Deanery of Postgraduate Medical and Dental Education, 20 Guilford Street, London WCIN 1DZ, UK; jfirth-cozens@

londondeanery.ac.uk

\section{REFERENCES}

1 Firth-Cozens J. Interventions to improve physicians' well being and patient care. Soc Sci Med 2001;52:215-22.

2 Sparks K, Cooper C, Fried Y, et al. The effects of hours of work on health: a metaanalytic review. J Occup Org Psychol 1997;70:391-408.

3 Kawakami N, Haratani T. Epidemiology of job stress and health in Japan: review of current evidence and future direction. Ind Health 1999;37:174-86.

4 Hobson J, Beach, JR. An investigation of the relationship between psychological health and workload amongst managers. Occup Med Lond 2000:50:518-22.

5 Firth-Cozens J. Individual and organizational predictors of depression in GP's. Br J Gen Pract 1998;48: 1647-51

6 Karasek R, Theorell T. Healthy work: stress, productivity and the reconstruction of working life. New York: Basic Books, 1990:89-103.

7 Spurgeon P, Barvel F, Maxwell R. Types of work stress and implications for the role of General Practitioners. Health Serv Manage Res 1995;8:186

8 Firth-Cozens J, Greenhalgh J. Doctors' perceptions of the links between stress and lowered clinical care. Soc Sci Med 1997;44:1017-22.

9 Foushee HC, Helmreich RL, Group interaction and flight crew performance. In: Weiner EL, Hagel DC, eds. Human factors in aviation. San Diego: Academic Press, 1988:189-227.

10 Firth-Cozens J, Moss F. Hours, sleep, teamwork and stress. BMJ 1998:317:1335-6.

11 Klazinga N, van Bolderen A. Epimetheus' responsibility: resident working hours and system redesign. Int J Qual Health Care 2003;15:109-10.
12 Kivimaki $M$, Sutinen $R$, Elovainion $M$, et al. Sickness absence in hospital physicians: 2 year follow-up study on determinants. Occup Environ Med 2001;58:361-6.

13 Firth-Cozens J. Cultures for improving patient safety through learning: the role of teamwork Qual Health Care 2001; 10(Suppl II): ii26-31.

14 Wesnes K, Walker M, Walker L. Cognitive performance and mood after a weekend on call in a surgical unit. $\mathrm{Br} J$ Surg 1997;84:493-5.

15 Smith L, Folkard S, Poole CJM. Increased injuries on night shift. Lancet 1994;344:1137-9.

16 Weinger M B, Ancoli-Israel S. Sleep deprivation and clinical performance. JAMA 2002;287:955-7.

17 Pilcher JJ, Huffcutt A. Sleep deprivation on performance: a meta-analysis. Sleep 1996;19:318-26.

18 Taffinder NJ, McManus IC, Gul Y, et al. Effect of sleep deprivation on surgeons' dexterity on laparoscopy simulator. Lancet 1998:352:1191.

19 Grantcharov TP, Bardram L, Finch-Jensen P. Performance after one night on call in a surgical department: Prospective study. BMJ 2001;323:1222-3.

20 Howard SK, Gaba DM, Rosekind RM, et al. Excessive daytime sleepiness in resident physicians: risks, intervention and implication. Acad Med 2002;77:1019-25.

21 Takahashi M, Nakata A, Haratani T, et al. Postlunch nap at worksite to promote daytime alertness in factory workers. J Sleep Res 2004 (in press).

22 Frey R, Decker K, Reinfried L, et al. Effect of rest on physicians' performance in an emergency department, objectified by electroencephalographic analyses and psychometric tests. Neurol Crit Care 2002;30:2322-9.

\section{Knowledge and action in stroke-are either good enough?}

\section{K M McPherson, P Kersten}

\section{It is important that education on stroke is provided in ways that people can really understand}

G etting people to hospital quickly for specialist care after a stroke saves lives and reduces disability. ${ }^{1}$ However, for many people, the most appropriate care is delayed and, in some cases, may not happen at all. Other than the ongoing lack of specialist stroke services, what is it that impedes access to best services for patients who have a stroke?

Huge efforts have been made to highlight the seriousness of stroke and the importance of getting swift medical attention. However, the long lasting effects of education and screening campaigns are debatable, ${ }^{2-4}$ providing a reminder (if one were needed) of the complexity of health promotion for behaviour change. Key questions to face are whether the level of knowledge about stroke is good enough, and whether that knowledge leads to appropriate action when people experience a stroke.

A number of recent studies have explored the quality of the public's knowledge about stroke and many make depressing reading. ${ }^{23}$ The recent study by Carroll et $a l^{5}$ (summarised in the "Echo" which appears on page 168 of this issue of $Q S H C$ ) presents contrasting findings about the level of knowledge, and prompts some interesting questions about what we do in relation to that knowledge that is important for practitioners, researchers, and policy makers alike. On the positive side, Carroll et $a l^{5}$ found a "good" level of knowledge among four groups of people (comprising patients in hospital after a stroke, those at risk of stroke from specialist clinics, members of the public who were visitors to other wards of the hospital, and nurses on general medical wards) in comparison with other studies. The majority knew at least one symptom of stroke and considered the event a medical emergency. Interestingly, hospital nurses were the only group who did not consider it to be an emergency, although this may result from their frame of reference (an acute medical ward) leading to a tighter definition of medical emergency such as cardiac/respiratory arrest. It would be interesting to know how primary care health professionals in the areaincluding district nurses, health visitors and general practitioners-would answer the same questions, given their role in the community to advise people who are at risk of or experience a stroke. Certainly, a recent study of general practitioners found that the majority considered stroke an emergency and would call an ambulance. ${ }^{6}$ Most participants in the study by Carroll et al stated that they too would call an ambulance for assistance. However, the reality of what actually happened was different, with those in the stroke group calling their general practitioner or seeking help from a family member to do so. 
The apparent conflict between what people say they would do and what they actually do is intriguing. On the one hand, it is not unusual for peopleincluding probably most of us-to make plans for actions that are then not carried out, particularly in new and stressful circumstances. Along with this general caveat, it may also be that factors such as severity and type of stroke are important mediators of action taken. One could imagine that severe signs of stroke such as unconsciousness, hemiparesis, or aphasia might be more likely to prompt a call for an emergency ambulance than less obvious or transient symptoms including visual disturbance, weakness in a limb, or slurring of speech. Certainly, people who have had a stroke have reported not recognising the symptoms they experience because they were so different from those they had read about. ${ }^{7}$ Furthermore, if a diagnosis of stroke is not always straightforward for qualified medical practitioners, ${ }^{8}$ it is perhaps not surprising that patients themselves have difficulty in self-diagnosis or are reluctant to call out emergency services. It would seem that, in order to design and target education more effectively, further exploration concerning the messages people actually hear about stroke from the information given is needed.

The title of Carroll et al's paper states that "knowledge was good but action was poor", yet the figures for recognition of risk factors, particularly among the health professional group, seem low (median of 2 for nurses compared with a median of 1 for the other groups). This level of knowledge is surely not good enough, given that these were staff in medical wards (albeit not specialist neurology wards) and that a number of important and modifiable risk factors such as atrial fibrillation were rarely mentioned. As for the people who actually had risk factors, only one third could recall having been advised of that risk. Interestingly, the investigation by Sug Yoon et al found that those who recalled being advised about their risk of having a stroke were actually no better at recognising their own symptoms.

So where does this leave us when thinking about and targeting education on risk factors and their relation to stroke? Given that stroke remains a common and poorly understood condition, ${ }^{10}$ education for the general population and health professionals, as well as those with risk factors, is vital. There is no doubt that advances have been made in understanding the importance of the content, context and timing of "information giving" to enhance knowledge. However, while knowledge is power in some domains of life, catalysts to action are clearly less well understood in the case of stroke. It is important that we provide educationwhether for at risk groups or the general population-in ways that people can really understand and, indeed, act upon the knowledge gained. Simply providing "more" education is not an adequate response.

With the continued efforts of organisations such as the Stroke Association and others, increased prioritisation of research into stroke appears to be happening, at least in the UK. Along with other research priorities, how people make potentially life and death decisions when they have a stroke is clearly worth more detailed investigation if we are to provide timely appropriate services and improve our record in stroke care.
Qual Sat Health Care 2004:13:166-167. doi: 10.1136/qshc.2004.010504

\section{Authors' affiliations}

K M McPherson, School of Health Professions and Rehabilitation Sciences, University of Southampton, Southampton SOI7 1BJ, UK P Kersten, School of Medicine, University of Southampton, Southampton SOI7 1BJ, UK

Correspondence to: Dr K M McPherson, School of Health Professions and Rehabilitation Sciences, University of Southampton, Southampton SO17 1BJ, UK;

k.mcpherson@soton.ac.uk

\section{REFERENCES}

1 Stroke Unit Trialists Collaboration. Organised inpatient (stroke unit) care for stroke. Cochrane Database of Systematic Reviews 2002; 1:CD000197.

2 Greenlund KJ, Neff $\sqcup$, Zheng ZJ, et al. Low public recognition of major stroke symptoms. Am J Prevent Med 2003;25:315-9.

3 Delemos CD, Atkinson RP, Croopnick SL, et al. How effective are "community" stroke screening programs at improving stroke knowledge and prevention practices? Results of a 3-month followup study. Stroke 2003;34:e247-9.

4 Silver FL, Rubini F, Black D, et al. Advertising strategies to increase public knowledge of the warning signs of stroke. Stroke 2003;34:1965-8.

5 Carroll C, Hobart J, Fox C, et al. Stroke in Devon: knowledge was good, but action was poor. J Neurol Neurosurg Psychiatry 2004;75:567-71.

6 Middleton S, Sharpe D, Harris J, et al. Case scenarios to assess Australian general practitioners' understanding of stroke diagnosis, management, and prevention. Stroke 2003:34:2681-6.

7 Sug Yoon S, Heller RF, Levi C, et al. Knowledge and perception about stroke among an Australian urban population. BMC Public Health 2001;1:14.

8 Harbison J, Hossain O, Jenkinson D, et al. Diagnostic accuracy of stroke referrals from primary care, emergency room physicians, and ambulance staff using the face arm speech test. Stroke 2003;34:71-6.

9 Becker K, Fruin M, Gooding T, et al. Communitybased education improves stroke knowledge. Cerebrovasc Dis 2001;11:34-43.

10 Gompertz P, Slack A, Vogel M, et al. Education in stroke: strategies to improve stroke patient care. Hosp Med 2002;63:408-11. 